\title{
Determination of flatness from straightness measurements and characterization of the surface by four parameters
}

\author{
J. Meijer and W. de Bruin*
}

Since for medium and larger objects there is no usable flatness reference in the sub-micron range, flatness deviations must be found from straightness measurements. The extensive task of coupling the straight reference lines into a reference flat is done using a computer. This simplifies the measuring procedure and offers much statistical information. The method described can be seen as a generalization of the classical 'Union Jack' method. After evaluation, the measured surfaces are described by four independent characteristic parameters having an important practical meaning

For practical reasons, an equidistant rectangular grid is preferable. By measuring in two directions, the reference lines can be coupled at each grid point as shown in Fig 1 without giving a flat reference plane. In general, this plane contains torsion, however, with additional diagonal measurements, the reference plane will become flat. The method as described in this article has been based on a system of reference lines as shown in Fig 2. In this figure, all possible measurements are given, but not all measurements are necessary. If required, some directions or some points can be skipped making it possible to measure plates with any contours (Fig 5).

When three or more measurements are available at a gridpoint, outliers can be detected and eliminated automatically. It is preferable to make, where possible, all four measurements per gridpoint for the best accuracy and reliability. During the measurements, no attention should be paid to the vertical position and tilt of the reference lines, so the measuring speed can be high.

\section{Transformation of the reference lines into the reference plane}

Fig 3 shows the real surface, the coordinate system, one reference line $r$ and an arbitrary chosen reference plane $R$. The measured height $H_{r i j}$ contains an exact part and a stochastic part, the measuring error $\epsilon$. From Fig 3

$$
\epsilon_{r i j}=H_{r i j}-h_{i j}-a_{r}-j \alpha_{r}
$$

For the other directions of the reference lines similar equations are obtained. The unknown values of $h, a$ and $\alpha$ will be chosen in such a way that $\Sigma_{r i j} \epsilon^{2}$ is minimal. If the errors $\epsilon$ are normally distributed then

$$
S=\sum_{\substack{k=1 . . w \\ r=1 . . w \\ i=0 . . m \\ j=0 . . n}}\left(\epsilon_{k}-\epsilon_{r}\right)_{i j}{ }^{2}
$$

"Twente University of Technology, Enschede, The Netherlands

\section{Nomenclature}

B Base plane containing sphericity and torsion

$F$ Degrees of freedom

$H_{\text {rij }}$ Height to reference line $\mathrm{r}$

$M$ Coefficients matrix

$N$ Number of unknown reference line parameters

$P \quad$ Total number of measured grid points

$R \quad$ Reference plane, remaining rms

$S \quad$ Square sum of errors

$S_{i j} \quad$ Geometric term used for sphericity

$T_{i j}$ Geometric term used for torsion

$W$ Total number of measurements

$Z \quad$ Square sum of errors with respect to $B$

a Start height of reference line or regression plane

b Dimensionless bowrise, $\mu \mathrm{m} / \mathrm{m}$

b Known vector

d Distance to regression plane

$e_{x} \quad$ Grid mesh in $x$-direction

$e_{y} \quad$ Grid mesh in $y$-direction

$h_{i j}$ Height to reference plane $\mathrm{R}$

$h_{r i j}$ Height to reference line $\mathbf{r}$ (after transformation)

$i \quad$ Line number in $x$-direction

$j \quad$ Line number in $y$-direction

$k \quad$ Index number

I Index number

$m \quad$ Number of fields in $x$-direction

$n \quad$ Number of fields in $y$-direction

p Vector containing the parameters $a$ and $\alpha$

$r$ Reference line (direction)

$s \quad$ Estimator for $\sigma_{m}$

$s_{i j} \quad$ Height difference due to sphericity

$t \quad$ Bowrise

$w$ Number of measurements in one point

$z \quad$ Random number from $N\left(0, \sigma_{m}\right)$ generator

$\Delta_{\text {rij }}$ Difference from the local mean

$\alpha \quad$ Trend of a reference line or regression plane

$\beta \quad$ Trend of regression plane

$\delta \quad$ Height difference with respect to $B$

$\eta \quad$ Principal direction (extreme bowrise)

$\theta \quad$ Direction, clockwise from $x$-axis

$\rho$ Sphericity, $\mathrm{rad} / \mathrm{m}$

$\sigma_{b} \quad$ Standard deviation of bowrise

$\sigma_{m} \quad$ Standard deviation of measurements

$\sigma_{r} \quad$ Standard deviation of results

$\tau$ Torsion, $\mathrm{rad} / \mathrm{m}$

$\phi \quad$ Direction of torsion vector

$\psi \quad$ Direction of directrices 
becomes minimal. In this way, all heights $h_{i j}$ drop out, so $S$ contains as unknowns only the initial heights $a$ and the tilts $\alpha$ of the reference lines. Three unknowns can be chosen freely by fixing the position of the reference plane $R$. Then for a complete grid, the remaining number of unknowns becomes:

$$
N=6(m+n)-11
$$

The sum $S$ (Eq (2)) will be minimal if, for all $N$ unknowns,

$$
\frac{\partial S}{\partial a}=\frac{\partial S}{\partial \alpha}=0
$$

From this condition, $N$ linear equations are obtained. Ranging the unknowns in the vector $p$ and the contributions of the measurements in the known vector $b$ this set of equations can be written as:

$$
\left[\begin{array}{ccccc}
m_{11} & \ldots & m_{1 l} & \ldots & m_{1 N} \\
\vdots & & \vdots & & \vdots \\
m_{k 1} & \ldots & m_{k l} & \ldots & m_{k N} \\
\vdots & & \vdots & & \vdots \\
m_{N 1} \ldots & \ldots & m_{N 1} & \ldots & m_{N N}
\end{array}\right]\left[\begin{array}{c}
p_{1} \\
\vdots \\
p_{k} \\
\vdots \\
p_{N}
\end{array}\right]=\left[\begin{array}{c}
b_{1} \\
\vdots \\
b_{k} \\
\vdots \\
b_{N}
\end{array}\right]
$$

or, shorter, $M p=b$

Because $S$ forms a sum over all points $P_{i j}$, the differentiation can be done point after point. By differentiation to a certain parameter $p_{k}$, only those terms of $S$ containing $p_{k}$ are of interest. When $w$ measurements are made in one gridpoint, this delivers $2 w$ contributions to the matrix $M$ and $w$ contributions to the vector $b$. After starting with empty $M$ and $b$ they are filled by adding the results of the point after point differentiation.

The indices $k$ and / in Eq (4) are computed as functions of the coordinates $i$ and $j$, the grid dimensions $n$ and $m$, the direction $r$ of the reference line and type of parameter ( $a$ or $\alpha$ ). This is done by short-named function procedures which give an unambiguous continuous indexing $^{1}$. If a certain reference line fails, then two parameters ( $a$ and $\alpha$ ) are missing, so two rows and coiumns remain empty, reducing the set of equations. After solution of Eq (4), all values of $a$ and $\alpha$ are known. From Eq (1)

$$
h_{r i j}=H_{r i j}-a_{r}-j \alpha_{r}
$$

The best approximation for the height $h_{i j}$ follows from

$$
\bar{h}_{i j}=\frac{1}{w} \sum_{r=1}^{w} h_{r i j}
$$

\section{Error analysis}

In each point the differences $\Delta_{r i j}=h_{r i j}-\bar{h}_{i j}$ from the mean value can be calculated. There are $F$ degrees of freedom given by

$$
F=W-N-P
$$

For a complete grid (Fig 2), $F=3(m n-m-n)+2$. The classical 'Union Jack' measurement, which should be seen as a special case of our method $(m=n=2)$ is based therefore on only two degrees of freedom. An unbiased estimator for the standard deviation $\sigma_{m}$ of the measurements is found from:

$$
s=\frac{1}{F}\left\{\sum_{1}^{W} \Delta_{r i j}^{2}\right\}^{1 / 2}
$$

The accuracy of $s$ depends on $F$. In practice, when $F>50$ the difference between $s$ and $\sigma_{m}$ may be neglected.

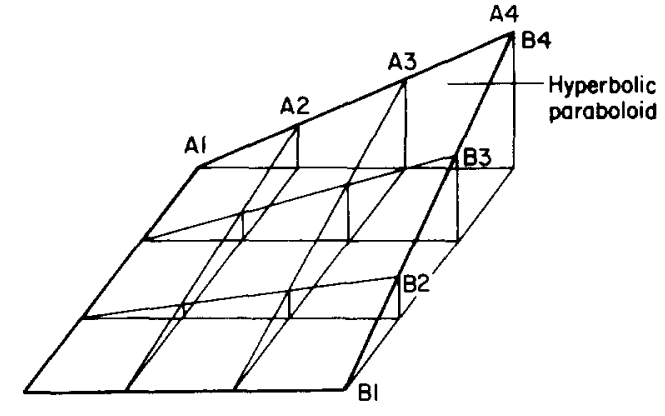

Fig 1 Two intersecting bundles of straight lines can form a hyperbolic paraboloid

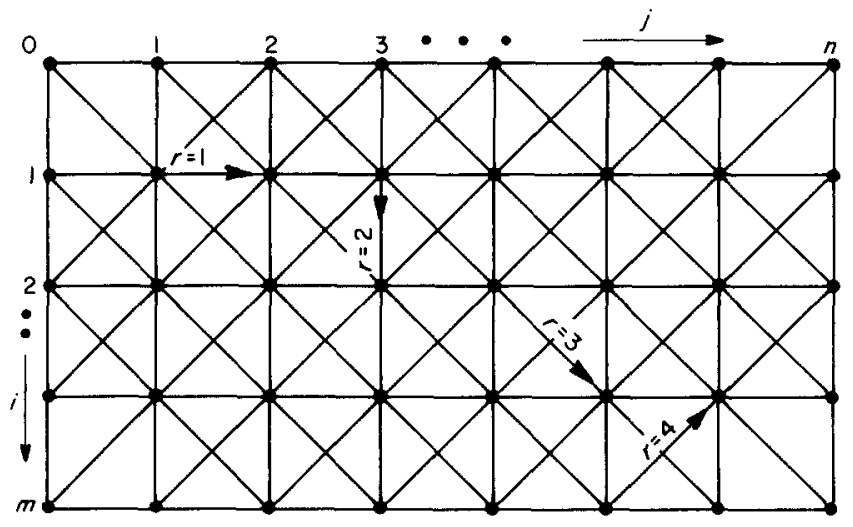

Fig 2 Applied grid. A height observation at the point i,j with respect to a reference line having direction $r$ is shown as $\mathrm{H}_{\text {rij }}$

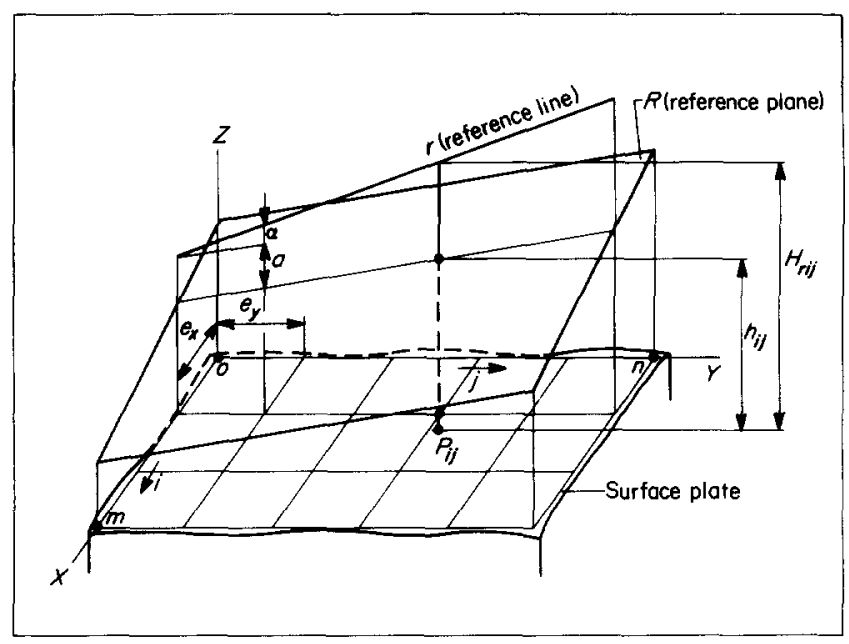

Fig 3 Coordinates, reference line and reference plane

Measurements $H_{r i j}$ giving a difference $\Delta_{r i j}>4 s$ are unreliable and will be eliminated by replacing them by the symbol *. After that elimination procedure, the computation is repeated. Because the mean value $\bar{h}_{i j}$ will be influenced by the outlier the test is made from the median. To recognize an outlier at least three measurements per grid point are necessary. The main importance of the test is to be sure that there are no outliers. From the last 50 surface plate calibrations as described by de Bruin ${ }^{4}$ only eight outliers have been detected and eliminated. 


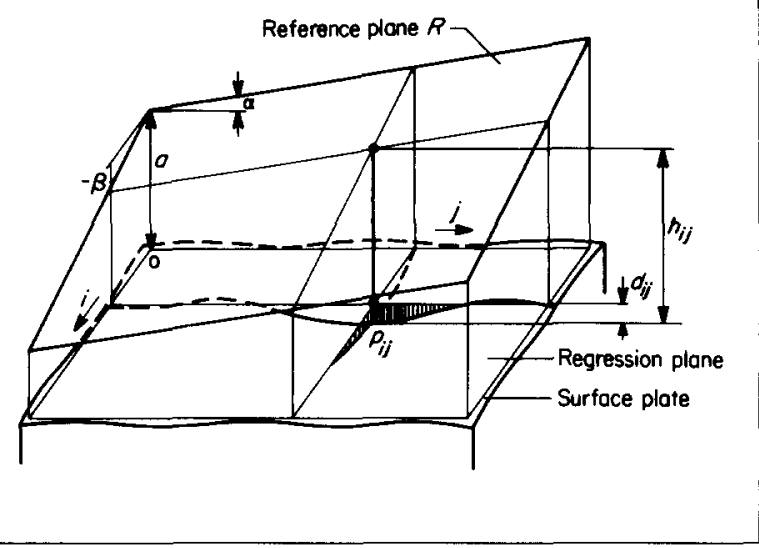

Fig 4 Relation between surface plate, regression plane and reference plane. The regression plane is chosen so that the square sum of the distances $d$ is minimal

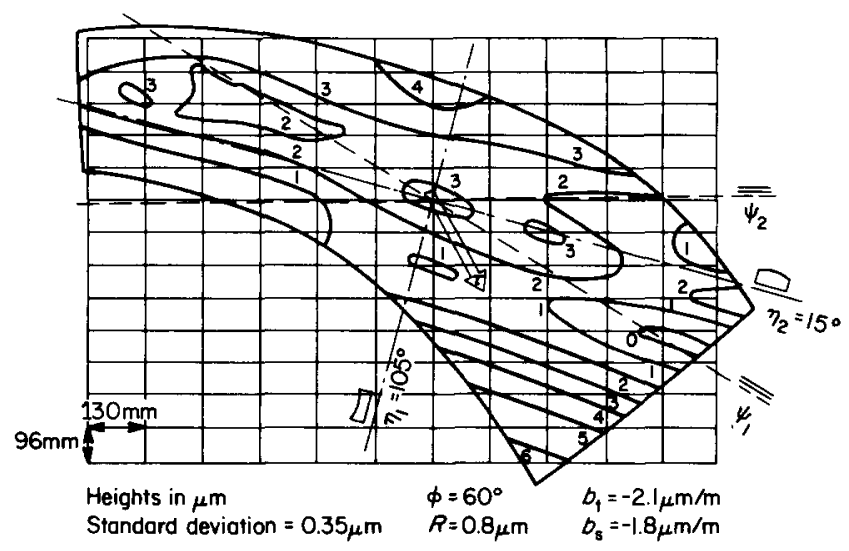

Fig 5 Polar piece. Grid dimensions $1430 \times 1248 \mathrm{~mm}$. Measured by order of the institute for nuclear physics, Amsterdam (1978)

\section{Input data and presentation of the results}

The first input data are about the grid pattern $m, n, e_{x}$ and $e_{y}$ so the programme can define space for the measurements by a matrix $H[1: 4,0: m, 0: n]$. The measurements should be read in strict numerical order. If a measurement fails, the symbol * should be read on that place. This basic information is sufficient to do the computations. All other information can be derived from this basic information.

If, for instance, only one height at a point has been measured even this single value will be removed because Eq (2) needs at least two measured heights per point. From the number of * symbols the real number of measurements $W$ and grid points $P$ are determined. The size of the matrix $M$ is initiated by the maximum value of $N$ following Eq (3), but after allowing for eventually empty lines and rows $N$ is replaced by the actual number of reference line parameters after which the actual value of $F$ is also computed. Note that after eliminating an outlier $F$ will be decreased.

\section{The regression plane}

After the transformation procedure, the heights $h_{i j}$ are given with respect to an arbitrary chosen reference plane $R$. To get easily comparable results the regression plane has been chosen as a new basis. The relation between the heights $h$ from the reference plane $R$ and the distances $d$ to the regression plane is given by $\mathrm{Eq}(8)$ and $\mathrm{Fig} 4$.

$$
d_{i j}=h_{i j}-a-j \alpha-i \beta
$$

For the regression plane $S=\Sigma d^{2}$ should be minimal. Then, the parameters $a, \alpha$ and $\beta$ are found from:

$$
\frac{\partial S}{\partial a}=\frac{\partial S}{\partial \alpha}=\frac{\partial S}{\partial \beta}=0
$$

or

$$
\left[\begin{array}{l}
a \\
\alpha \\
\beta
\end{array}\right]=\left[\begin{array}{ccc}
\Sigma 1 & \Sigma j & \Sigma i \\
\Sigma j & \Sigma j^{2} & \Sigma i j \\
\Sigma i & \Sigma i j & \Sigma i^{2}
\end{array}\right]^{-1}\left[\begin{array}{l}
\Sigma h_{i j} \\
\Sigma j h_{i j} \\
\Sigma i h_{i j}
\end{array}\right]
$$

in which each summation $\Sigma$ is taken only over those gridpoints where a value of $h$ is available (measured points). The results can be given as a table of $d$-values (Eq (8)), but in most cases a contour map drawn by the computer is preferred. When the coordinates of the contour of the surface are given as additional input data, this contour will be drawn too (Fig 5 ).

\section{Characteristic values}

After several years of practice with surface plate calibrations the need was felt for some simple parameters to characterize the form of the surface. Parameters which have a clear technical meaning and which are fully independent are torsion and sphericity ${ }^{*}$. A third necessary parameter is the direction of the torsion axis (the torsion vector is a free vector and therefore not tied to any point). From these three parameters the main form of most surfaces could be reconstructed. Usually there remains a so-called stochastic part, including all higher order effects. To quantify this part a fourth parameter has been defined as the rms-value of the actual surface compared to the reconstructed surface. This value is called the 'remaining $\mathrm{rms}^{\prime}(R)$.

These four parameters have been shown to be useful for describing both the character as well as the quality of the measured surfaces. Changes of a surface plate as caused by temperature, humidity, support or wear could also be shown easily. In many cases, only one parameter is influenced (eg a temperature gradient influences only the sphericity, while wear affects the remaining $\mathrm{rms}$ ).

\section{Calculation of the characteristics}

The magnitude of torsion and sphericity is found from regression analysis with respect to a base plane $B$ which contains the parameters $a, \alpha$ and $\beta$ (Fig 4) and torsion $(\tau)$ and sphericity $(\rho)$ as free parameters. This plane can be seen as a superposition of a flat, a spherical and a twisted plane. A height difference $s_{i j}$ due only to sphericity (Fig 6)

\footnotetext{
* It would be obvious to describe the surface mathematically by a quadric $\left(\mathrm{z}=a \mathrm{x}^{2}+b \mathrm{y}^{2}+c \mathrm{xy}\right)$, ranging all higher order terms in a complementary macro roughness. In a quadric surface, there exist two principal normal sections respectively associated with the largest value $\rho_{1}$ and the smallest value $\rho_{2}$ of the curvature (the principal curvatures of the surfacel. The planes of the principal normal sections are mutually perpendicular. Instead of the principal curvatures, the mean curvature $1 / 2\left(\rho_{1}+\rho_{2}\right)$ and the difference $1 / 2\left(\rho_{1}-\rho_{2}\right)$ can be used to describe the surface. For our application, the latter description, namely splitting sphericity and torsion, is in principle the same, but offers some physical advantages, because there are phenomena which influence only one of these.
} 
can be expressed by

$$
s_{i j}=\rho S_{i j}
$$

where

$$
S_{i j}=-1 / 2\left(x_{i}^{2}+y_{j}^{2}\right)
$$

Torsion may be expressed by a free vector, which can be tied to any point. Taking the origin 0 then the height dif ference $t_{i j}$ due to real torsion (Fig 7) can be expressed by

$$
t_{i j}=\tau T_{i j}
$$

where

$$
T_{i j}=1 / 2\left(y_{j}{ }^{2}-x_{i}{ }^{2}\right) \sin 2 \phi-x_{i} y_{j} \cos 2 \phi
$$

Similarly to Eq (8), the height difference $\delta$ from the measured surface to the new base $B$ can be expressed by

$$
\delta_{i j}=h_{i j}-a-j \alpha-i \beta-T_{i j} \tau-S_{i j} \rho
$$

From the condition $Z=\Sigma \delta^{2}$ is minimal, the parameters $a$, $\alpha, \beta, \tau$ and $\rho$ can be found from

$$
\frac{\partial Z}{\partial a}=\frac{\partial Z}{\partial \alpha}=\frac{\partial Z}{\partial \beta}=\frac{\partial Z}{\partial \tau}=\frac{\partial Z}{\partial \rho}=0
$$

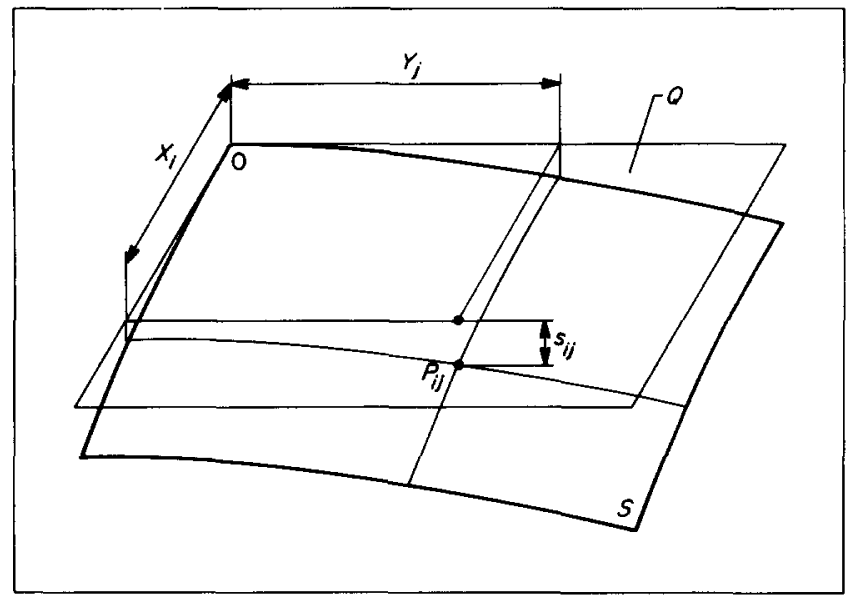

Fig 6 Height difference due to sphericity

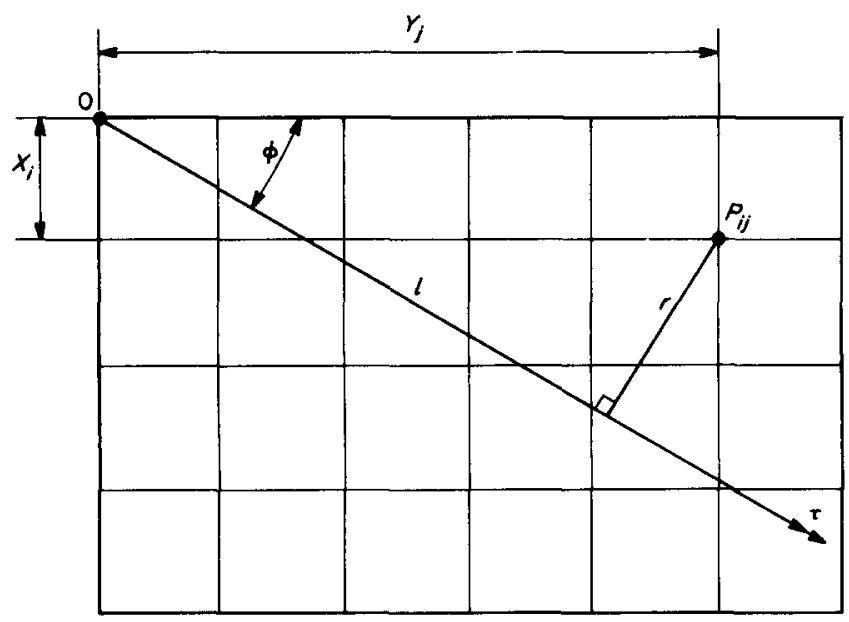

Fig 7 Height difference due to torsion

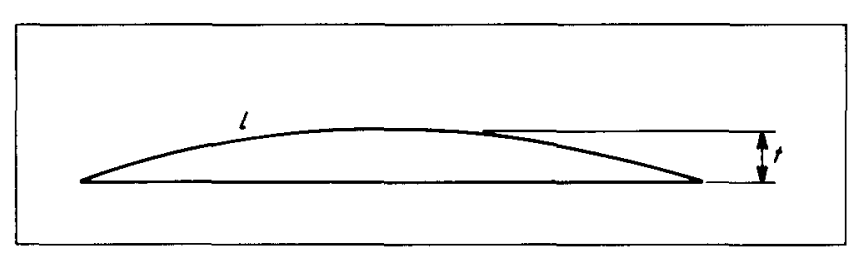

Fig 8 The dimensionless bowrise is defined by $\mathrm{b}=\mathrm{t} / \mathrm{l}$ or

$$
\left[\begin{array}{l}
a \\
\alpha \\
\beta \\
\tau \\
\rho
\end{array}\right]=\left[\begin{array}{ccccc}
\Sigma 1 & \Sigma j & \Sigma i & \Sigma T & \Sigma S \\
\Sigma j & \Sigma j^{2} & \Sigma i j & \Sigma j T & \Sigma j S \\
\Sigma i & \Sigma i j & \Sigma i^{2} & \Sigma i T & \Sigma i S \\
\Sigma T & \Sigma j T & \Sigma i T & \Sigma T^{2} & \Sigma T S \\
\Sigma S & \Sigma j S & \Sigma i S & \Sigma T S & \Sigma S^{2}
\end{array}\right]^{-1}\left[\begin{array}{c}
\Sigma h \\
\Sigma i h \\
\Sigma i h \\
\Sigma T h \\
\Sigma S h
\end{array}\right]
$$

The parameter $\phi$, which does not follow directly from this equation, is found in an iterative way. After starting the procedure with an initial value $\phi_{0}$ the other corresponding parameters are obtained from $\mathrm{Eq}(13)$ and the function $Z=Z(\phi)$ can be calculated. This function is minimized by stepwise variations of $\phi$ converging to the final solution. Then the last parameter, the remaining rms, can be found from

$$
R=\left(\frac{Z}{P-6}\right)^{1 / 2}
$$

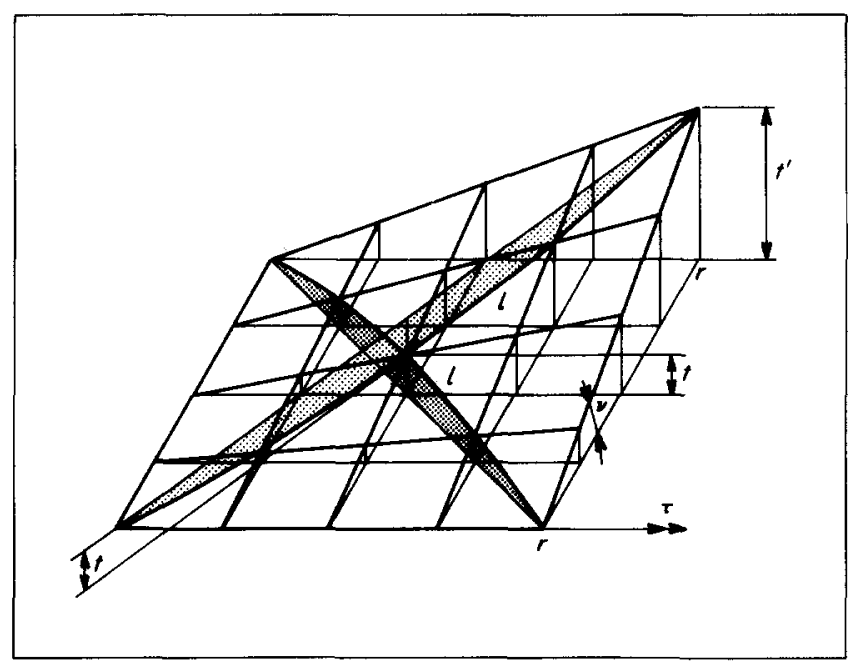

Fig 9 Bowrise $\mathrm{t}$ in a surface with real torsion

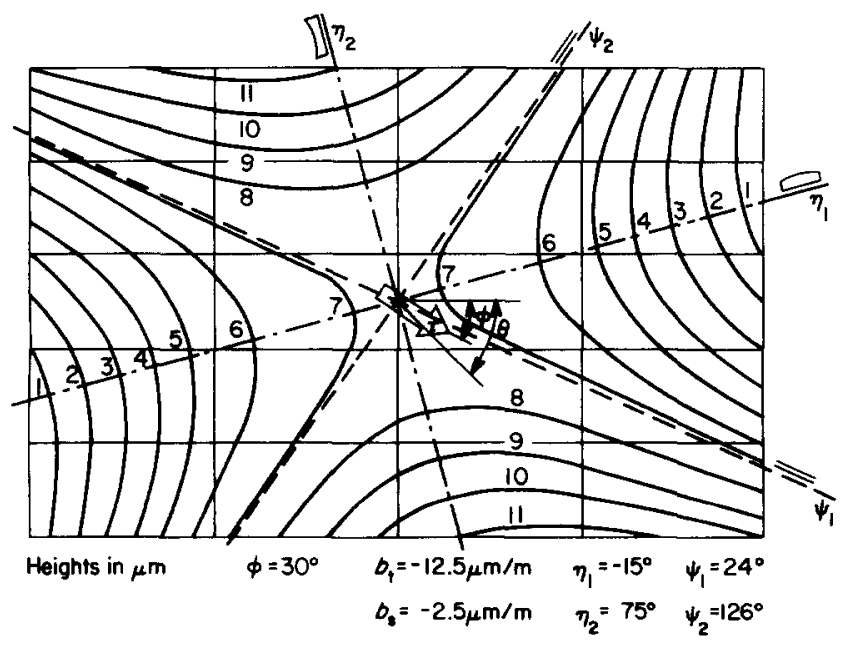

Fig 10 Map of a surface with torsion and sphericity. The principal directions $\eta_{1}$ and $\eta_{2}$ are mutually perpendicular and are found in directions $\phi \pm 45^{\circ}$. All lines with the direction of the directrices $\psi$ are straight lines in the surface 


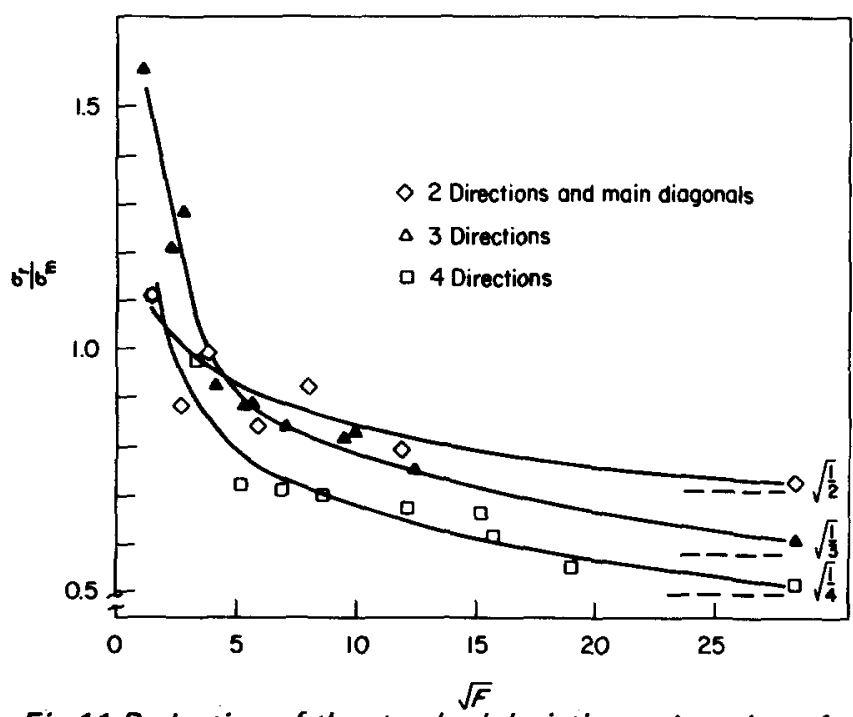

Fig 11 Reduction of the standard deviation as function of the number of measurements per point and the number of degrees of freedom

\section{Principal directions in the surface}

There are some advantages in expressing both sphericity and torsion in a dimensionless bowrise $b=t / /$ (Fig 8). Due to sphericity this value will be

$$
b_{s}=\frac{1}{8} \rho l
$$

where $1 / \rho$ represents the radius of curvature. In a surface containing only torsion, bowrise can be found. Extreme values are found in the directions $\phi \pm 45^{\circ}$. From Fig 9 $t=1 / 4 t^{\prime}$ where $t^{\prime}=1 / 2 \tau /^{2}$ can be seen.

Extreme values of dimensionless bowrise due to torsion are obtained from

$$
b_{t}=\frac{1}{8} \tau \prime
$$

In any arbitrary direction $\theta$ (Fig 10) the bowrise due to torsion is

$$
b_{t, \theta}=b_{t} \sin 2(\theta-\phi)
$$

So the total dimensionless bowrise in a direction $\theta$ amounts to

$$
b_{\theta}=b_{s}+b_{t} \sin 2(\theta-\phi)
$$

Directions giving extreme values of $b_{\theta}$ are the principal or main directions and are found to be

$$
\begin{aligned}
& \eta_{1}=\phi+45^{\circ} \operatorname{sign}\left(p_{t}\right) \quad(\max ) \\
& \eta_{2}=\phi-45^{\circ} \operatorname{sign}\left(p_{t}\right) \quad \text { (min) }
\end{aligned}
$$

If abs $\left(b_{s} / b_{t}\right)<1$ there will be also directions with $b_{\theta}=0$ (straight lines or directrices). The surface is a saddle plane or hyperbolic paraboloid. These directions can be found from

$$
\begin{aligned}
& \psi_{1}=\phi+1 / 2 \arcsin \left(-b_{s} / b_{t}\right) \\
& \psi_{2}=\phi+1 / 2\left[180^{\circ}-\arcsin \left(-b_{s} / b_{t}\right)\right]
\end{aligned}
$$

A special case is given by $\left|b_{s}\right|=\left|b_{t}\right|$. Then the surface is a cylinder with both directrices coinciding with one principal direction. Finally, if $\left|b_{s} / b_{t}\right|>1$ the surface is part of an ellipsoid or a sphere $\left(b_{t}=0\right)$.

\section{Accuracy and significance of the results}

In the case of normally distributed measuring errors the standard deviation of the measurements is obtained by Eq (7). The heights as mentioned in the measuring report

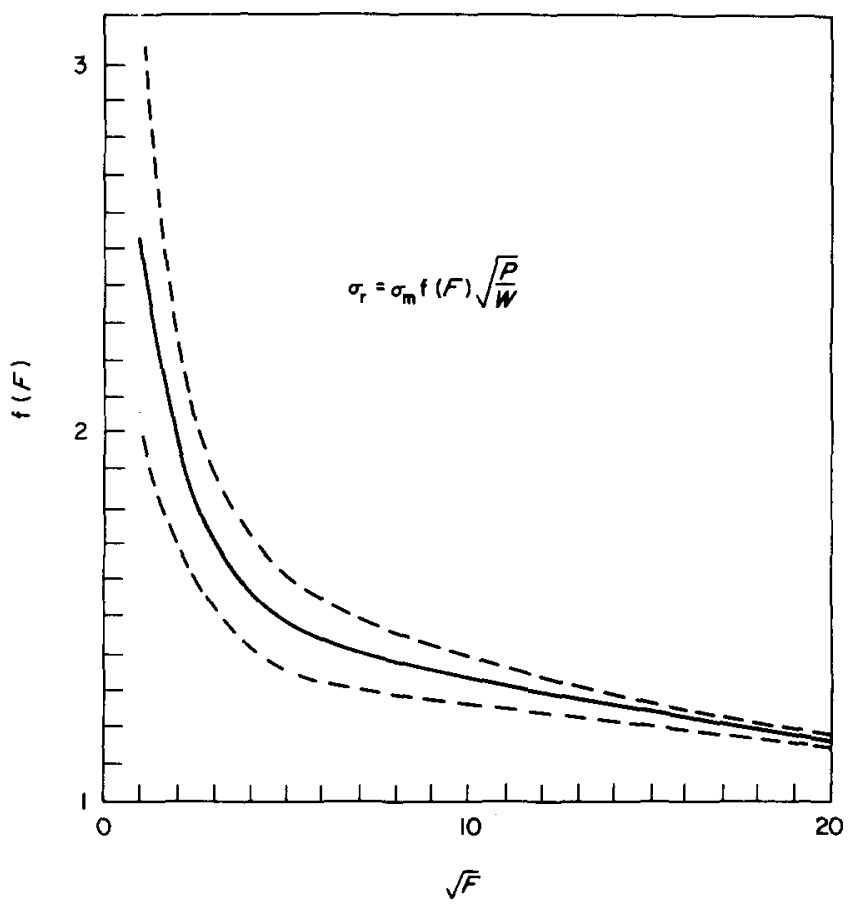

Fig 12 More degrees of freedom give a better accuracy of the results

are the means of $w$ values per point, so the standard deviations will be reduced by $w^{-1 / 2}$. In general, with $W$ observations over $P$ points the reduction factor will be $P / W^{1 / 2}$. Up to now, the computed values of $a$ and $\alpha$ are assumed to be exact, but in reality there are small deviations enlarging the deviations of the calculated heights by a factor which will be a function of $F$ (degrees of freedom). The reduction factor $\sigma_{r} / \sigma_{m}$ has been found from simulations with known $\sigma_{m}$. The results are shown in Fig 11. Distinction has been made between grids completely measured in four directions, grids measured in only three directions and grids measured in only two directions ( $x$ and $y$ ) completed with both main diagonals. A special case forms the 'Union Jack' pattern, which belongs both to the first as well as to the last collection. For a very large grid, the curves will be near to the asymptotic value $k^{\prime-1 / 2}$ or in general $P W^{1 / 2}$. This systematic effect can be separated by

$$
\sigma_{r}=\sigma_{m} f(F)\left(\frac{P}{W}\right)^{1 / 2}
$$

The factor $f(F)$, caused by the flexibility of the reference lines, can be expressed by one curve (Fig 12). This figure is valid for arbitrary grids and numbers of measurements and makes it possible to find the value of $\sigma_{r}$ because after each computer evaluation values of $\sigma_{m}, P$ and $W$ are known. The broken side lines in Fig 12 represent the one standard deviation boundaries which could be calculated too because each simulation has been repeated ten times.

It is very interesting to know whether the characteristic values are significant. It should be known which part of these values are the cause of the measuring errors given by $\sigma_{m}$. In the simulations as mentioned above the simulated heights are obtained from a $N\left(0, \sigma_{m}\right)$ generator delivering normal distributed numbers. In this case there is no real torsion or sphericity so the calculated values of $b_{t}$, $b_{s}$ and $R$ are the stochastic parts directly. The expected values of $b_{t}$ and $b_{s}$ are zero, so only the standard deviation has been calculated. Values of $\sigma_{b} / \sigma_{m}$ are shown in Figs 13 and 14. In real cases when torsion and sphericity will occur this ratio will be the same, then the functions 
as given in Figs 13 and 14 are used to find $\sigma_{b}$ from the calculated $\sigma_{m}$. In the measuring report these standard deviations are given in brackets after the values of $b_{t}$ and $b_{s}$. The remaining rms $R$ has been calculated from the simulations and has been found to be about $0.65 \sigma_{m}$.

\section{Interrelated height measurement}

The analysis as described before has been based on independent measurements of the heights $H_{r i j}$. Fortunately. there are a lot of methods for measuring in this way. The reference line can be a straight edge, a laser beam etc. However, there are also methods measuring the height difference from a point with respect to the former point on the same line, giving dependent measurements.

Because the last category of methods is also in use, the difference between both methods has been analysed. In both cases a flat surface was simulated by $N\left(0, \sigma_{m}\right)$ distributed numbers $z$. In the first case, the heights $H_{r i j}$ were equalled to the generated value of $z$, while in the second case the first height on each line was put at zero, while in all following points the height was found from the former point and the simulated 'height difference' $z$. In both cases the standard deviation of the results $\sigma_{r}$ was calculated based on the same set of random numbers $z$. From the results of 16 simulations with different grid dimensions no significant difference could be found. A method of the second group has been described in more detail $^{4}$ showing the importance of the characteristics.

\section{Conclusions}

An evaluation method for flatness measurements is available to obtain reliable values of flatness deviations in the sub-micron range. In this method a test for errors is made after which unreliable values can be removed without the need to measure them again.

Four independent characteristic parameters are defined to describe the functional properties of the surface. These values can be calculated easily from the results of the evaluation as described, but also from each other measuring result.

The authors aim to standardize these parameters in order to classify surface plates.

\section{Acknowledgement}

This project has been initiated and supported by Prof. dr.ir. A. Nawijn, who also gave many suggestions after reading this manuscript.

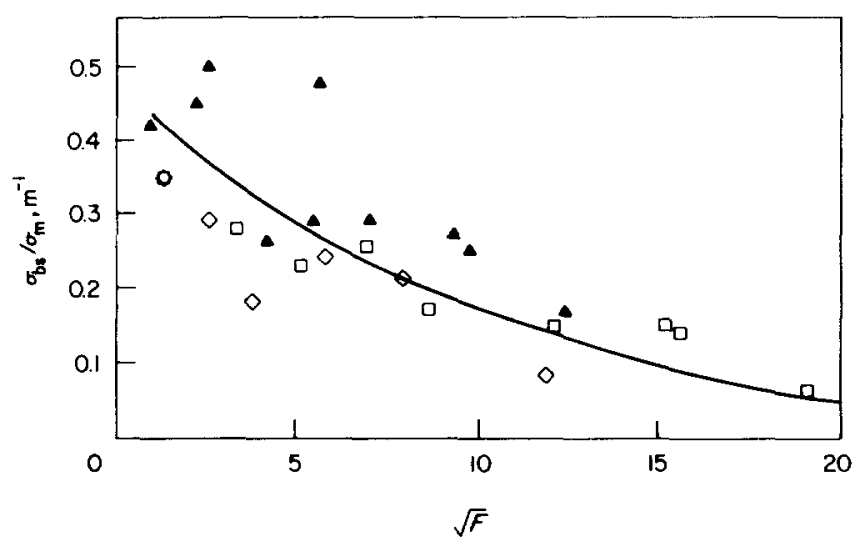

Fig 13 Standard deviation of the dimensionless bowrise due to sphericity

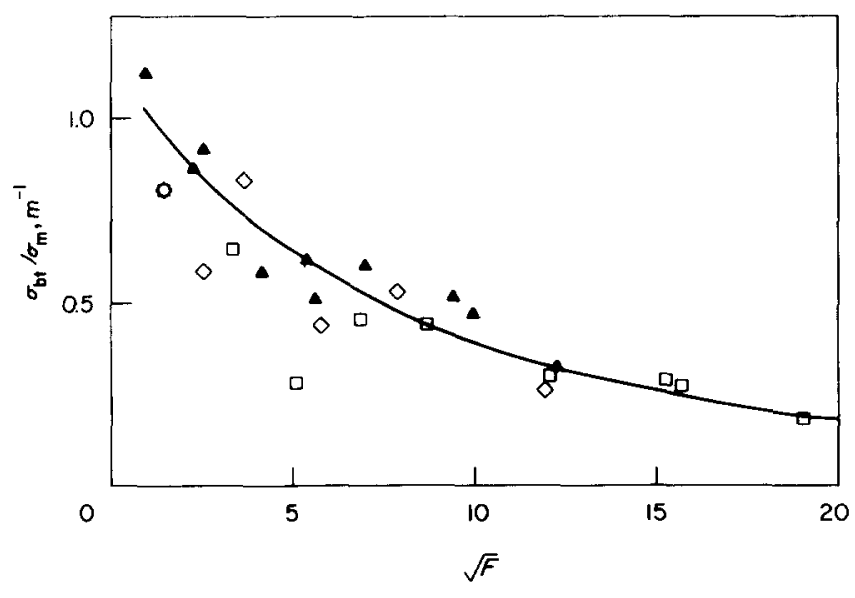

Fig 14 Standard deviation of the dimensionless bowrise due to torsion

\section{References}

1. Meijer J. A New Method for Calibrating Surface Plates. Mechanical Communications, 1975, No. 4, Twente University of Technology. Enschede, The Netherlands

2. de Bruin W., Meijer J. and Nawijn A. Surface Plate Measurements. Annals of the CIRP, 1977, 26(1), 251-256

3. de Bruin W. and Meijer J. Analysis of Flatness Measurement and Form Stability of a Granite Surface Plate. Annals of the CIRP. $1980,29(1), 385-390$

4. de Bruin W. and Meijer J. Some Results of Flatness Measurement. Precision Engineering, January 1981, 3(1), 23-26 